\title{
Survey on Product Review Sentiment Analysis with Aspect Ranking
}

\author{
Harsha Patil ${ }^{1}$, P. M. Mane ${ }^{2}$ \\ ${ }^{1}$ P.G. Scholar, Dept. of Computer Engineering, Zeal COE \& Research, Savitribai Phule University of Pune, India \\ ${ }^{2}$ Professor, Dept. of Computer Engineering, Zeal COE \& Research, Savitribai Phule University of Pune, India
}

\begin{abstract}
Millions of reviews on products are now available on internet. Consumers commonly seek for quality information from online customers review prior to make their purchasing product decision while many firms use online reviews as important feedbacks in their product development, marketing and consumer relationship management. Both consumers and firms are benefited by this rich and valuable knowledge from consumers review. When reviews on various aspects of the products are in textual format, it is difficult to identify and analyze such reviews so we are developing the system to mine those aspects and rank them which will help for better product development. We proposed product aspect ranking framework, which automatically identifies the important aspects of products from online consumer reviews, aiming at improving the usability of the numerous reviews. Important product aspects identification is based on two observations: 1) the important aspects are usually commented on by a large number of consumers and 2) overall opinions on the product is decided by opinions on important aspects of those products. In particular, consumer reviews of a product are given in textual format; we first parse the reviews with Natural Language Processor to identify the aspects of particular product then for sentiment analysis we use sentiment classifier such as Nä̈ve Bays or SVM to classify the comments as positive and negative sentiments. After sentiment analysis we apply Probabilistic aspect ranking algorithm to conclude the importance of aspects by simultaneously considering aspect frequency and the influence of consumer opinions given to each aspect over their overall opinions.
\end{abstract}

Keywords: consumer review, product aspects, aspect ranking, aspect identification, sentiment classification.

\section{Introduction}

In the recent years the use of e-commerce is grown very rapidly. Most retail Websites promotes consumers to write their feedbacks about products to express their opinions on various aspects of the products. An aspect, which can also be called as feature, refers to a component or an attribute of a certain product. Many forum Websites also provide a platform for consumers to post reviews on number of products. For Example, CNet.com involves more than seven million product reviews; These numerous consumer reviews contain rich and valuable knowledge, which is becoming an important resource for both consumers and firms [2]. Before purchasing a product, consumers commonly seek quality information from online reviews and firms can use these reviews as feedbacks for better product development, consumer relationship management and marketing.

The identification of important product aspects plays an essential role in improving the usability of reviews which is beneficial to both consumers and firms. Consumers can easily make purchasing decision by paying attention to the important aspects, while firms can focus on the improvement of product quality so that product reputation is enhanced. However, manual identification of important aspects is impractical. Therefore, an approach to automatically identify the important aspects is highly demanded. Motivated by the above observations, we made a survey on different techniques used to find important product aspects automatically from online consumer reviews

For the development of the any product it needs different review of customers and also for different customer's reviews on any products helps them for purchasing the product. There are numerous reviews on various aspects of the product; we are developing the system to mine those aspects and rank them which will help for better product development

The task of aspect selection becomes more challenging with the numerous of data the reviews have different types when reviews are in textual format in that case we first parse the reviews with natural language processor to identify the aspects of particular product then for sentiment analysis we use sentiment classifier such as Naïve Bayes or SVM to classify the comments as positive and negative sentiments. After sentiment analysis we apply probabilistic aspect ranking algorithm to conclude the importance of aspects by simultaneously considering aspect frequency and the influence of consumer opinions given to each aspect over their overall opinions.

In this paper we present the methodology and techniques used for the product aspect identification and product aspect classification in the and also illustrates the product aspect ranking.

\section{Literature Survey}

We introduce a stochastic graph-based method for computing relative importance of textual units for Natural Language Processing. We test the technique on the problem of Text Summarization (TS)[2]. Extractive TS relies on the concept of sentence salience to identify the most important sentences in a document or set of documents. Salience is typically defined in terms of the presence of particular important words or in terms of similarity to a centroid pseudo-sentence. We consider a new approach, Lex Rank, for computing sentence importance based on the concept of eigenvector 


\section{International Journal of Science and Research (IJSR) \\ ISSN (Online): 2319-7064}

Index Copernicus Value (2013): 6.14 | Impact Factor (2014): 5.611

centrality in a graph representation of sentences. In this model, a connectivity matrix based on intra-sentence cosine similarity is used as the adjacency matrix of the graph representation of sentences. Our system, based on Lex Rank ranked in first place in more than one task in the recent DUC 2004 evaluation. In this paper we present a detailed analysis of our approach and apply it to a larger data set including data from earlier DUC evaluations. We discuss several methods to compute centrality using the similarity graph. The results show that degree-based methods (including Lex Rank) outperform both centroid-based methods and other systems participating in DUC in most of the cases. Furthermore, the Lex Rank with threshold method outperforms the other degree-based techniques including continuous Lex Rank. We also show that our approach is quite insensitive to the noise in the data that may result from an imperfect topical clustering of documents.[2]

With the rapid growth of the Internet, the ability of users to create and publish content has created active electronic communities that provide a wealth of product information. However, the high volume of reviews that are typically published for a single product makes harder for individuals as well as manufacturers to locate the best reviews and understand the true underlying quality of a product. In this paper, we reexamine the impact of reviews on economic outcomes like product sales and see how different factors affect social outcomes such as their perceived usefulness. Our approach explores multiple aspects of review text, such as subjectivity levels, various measures of readability and extent of spelling errors to identify important text-based features. In addition, we also examine multiple reviewerlevel features such as average usefulness of past reviews and the self-disclosed identity measures of reviewers that are displayed next to a review. Our econometric analysis reveals that the extent of subjectivity, in formativeness, readability, and linguistic correctness in reviews matters in influencing sales and perceived usefulness. Reviews that have a mixture of objective, and highly subjective sentences are negatively associated with product sales, compared to reviews that tend to include only subjective or only objective information. However, such reviews are rated more informative (or helpful) by other users. By using Random Forest-based classifiers, we show that we can accurately predict [3] the impact of reviews on sales and their perceived usefulness. We examine the relative importance of the three broad feature categories: "reviewer-related" features, "review subjectivity" features, and "review readability" features, and find that using any of the three features sets results in a statistically equivalent performance as in the case of using all available features. This paper is the first study that integrates eco- nonmetric, text mining, and predictive modeling techniques toward a more complete analysis of the information captured by user-generated online reviews in order to estimate their helpfulness and economic impact.[3]

The existence of the World Wide Web has caused an information explosion. Readers are overloaded with lengthy text documents where a shorter [4] version would suffice. All computer users, be it professionals or novice users, are particularly affected by this predicament. There exists an urgent need for the discovery of knowledge embedded in digital documents. This paper intends to investigate techniques and methods used by researchers for automatic text summarization. Special attention is paid to Bio-inspired methods for text summarization.[4]

Merchants selling products on the Web often ask their customers to review the products that they have purchased and the associated services. As e-commerce is becoming more and more popular, the number of customer reviews that a product receives grows rapidly. For a popular product, the number of reviews can be in hundreds or even thousands. This makes it difficult [5] for a potential customer to read them to make an informed decision on whether to purchase the product. It also makes it difficult for the manufacturer of the product to keep track and to manage customer opinions. For the manufacturer, there are additional difficulties because many merchant sites may sell the same product and the manufacturer normally produces many kinds of products. In this research, we aim to mine and to summarize all the customer reviews of a product.[5] This summarization task is different from traditional text summarization because we only mine the features of the product on which the customers have expressed their opinions and whether the opinions are positive or negative. We do not summarize the reviews by selecting a subset or rewrite some of the original sentences from the reviews to capture the main points as in the classic text summarization. Our task is performed in three steps: (1) mining product features that have been commented on by customers; (2) identifying opinion sentences in each review and deciding whether each opinion sentence is positive or negative; (3) summarizing the results. This paper proposes several novel techniques to perform these tasks. Our experimental results using reviews of a number of products sold online demonstrate the effectiveness of the techniques.

We address the problem of analyzing multiple related opinions in a text. For instance, in a restaurant review such opinions may include food, ambience and service. We formulate this task as a multiple aspect ranking problem, where the goal is to produce a set of numerical scores, one for each aspect. We present an algorithm that jointly learns ranking models for individual aspects by modeling the dependencies between assigned ranks. This algorithm guides the prediction of individual rankers by analyzing metarelations between opinions, such as agreement and contrast. We prove that our agreement based joint model is more expressive than individual ranking models. Our empirical results further confirm the strength of the model: the algorithm provides significant improvement over both individual rankers and a state-of-the-art joint ranking model.

\section{Existing system}

A textual reviews are provided as input to product aspect ranking system, these review are analyzed by using shallow parser dependency for aspect identification.[1] These aspects are nothing but features of the respective product. After aspect identification again the reviews are analyzed for sentiments with sentimental classifiers to classify for positive and negative sentiments of respect reviews. According to these sentiments the aspects are classified as positive and

\section{Volume 4 Issue 12, December 2015}




\section{International Journal of Science and Research (IJSR) \\ ISSN (Online): 2319-7064}

Index Copernicus Value (2013): 6.14 | Impact Factor (2014): 5.611

negative. Later those aspects are arranged by using Probabilistic Aspect Ranking algorithm.[1]

Disadvantages of Existing System:

- The drawbacks of the existing systems are that sentiment analysis cannot provide accurate classification of positive and negative comments. Hence can introduce errors in aspect ranking.

- The reviews are disorganized, leading to difficulties in information navigation and knowledge acquisition.

- The frequency-based solution is not able to identify the truly important aspects of products which may lead to decrease in efficiency of the review.

\section{System Architecture}

In this paper, we are implementing product aspect ranking framework, which automatically identifies the important aspects of products from online consumer reviews, aiming at improving the usability of the numerous reviews [1]. Important product aspects identification is based on two observations: 1) the important aspects are usually commented on by a large number of consumers and 2) overall opinions on the product is decided by opinions on important aspects of those products. In particular, consumer reviews of a product are given in textual format; we first parse the reviews with natural language processor to identify the aspects of particular product then for sentiment analysis we use sentiment classifier such as Naïve Bays or SVM to classify the comments as positive and negative sentiments. After sentiment analysis we apply probabilistic aspect ranking algorithm for usual aspect ranking. [1]

The process of product aspect ranking consisting of three main Steps:

A.Aspect identification;

B. Sentiment classification on aspects

C. Product aspect ranking

Given the consumer reviews of a product, first identify the aspects in the reviews and then analyze these reviews to find consumer opinions on the aspects via a sentiment classifier and finally rank the product based on importance of aspect by taking into account aspect frequency and consumers' opinions given to each aspect over their overall opinions. [1]

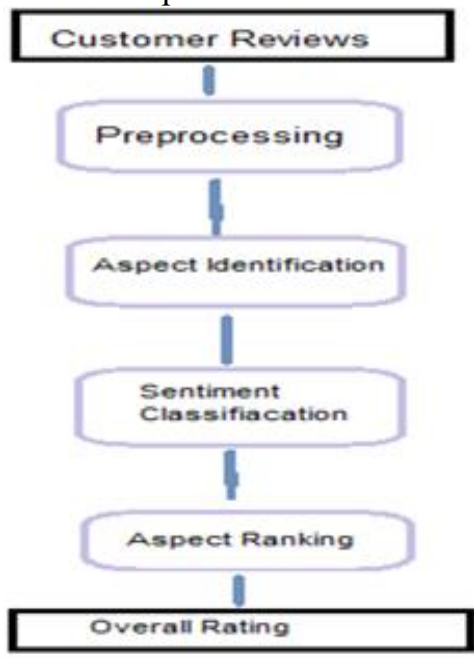

Figure 1: System Architectures.

\subsection{Product Aspect Identification Techniques:}

In the Pros and Cons reviews, the aspects are identified by extracting the frequent noun terms in the reviews. For identifying aspects in the free text reviews, first the free text reviews are spilt into sentences, each sentence is parsed using NLP Stanford parser. The frequent noun phrases are then extracted, with the help of above mentioned function, as candidate aspects.

Input: Pros and cons reviews and free text reviews Output: Product aspects

\subsection{Sentiment Classification on Reviews}

A Sentiment classifier is learned from the Pros reviews (positive reviews) and cons reviews (negative reviews). The classification is done using Naive Bayes model classifier or SVM classifier. The Pros and Cons reviews have explicitly categorized positive and negative opinions on the aspects. These reviews are valuable training samples for learning a sentiment classifier.

In the Supervised learning technique use the collection of labeled reviews to learn an extraction model. This extraction model called as extractor is then used for the identification of aspects in ne reviews. Most of the supervised learning techniques are based on the sequential learning

Input: Collection of reviews and identified aspects.

Output: The customer's opinion on specific aspects is found for each aspect.

\subsection{Product Aspect Ranking}

Aspect ranking algorithm calculates the weight of aspects of a product from consumer reviews. This algorithm uses the concept of TFIDF which is commonly used for calculation of weight of term in document. Here this concept is used for calculation of value of aspect term. Weight of aspect is calculated by using aspect value given by TFIDF and occurrence frequency of positively opinionated word and negatively opinionated words associated with aspect term.

Input: Collection of reviews and identified aspects.

Output: The customer's opinion on specific aspects is found for each aspect.

\section{Conclusion}

This survey paper presented an overview on the product aspect ranking techniques to identify important aspects of products. Product aspect ranking process contains three main steps i.e. product aspect identification, aspect sentiment classification and aspect ranking. We have conducted a survey which illustrates various methods for aspect identification and sentiment classification. 


\section{International Journal of Science and Research (IJSR) \\ ISSN (Online): 2319-7064}

Index Copernicus Value (2013): 6.14 | Impact Factor (2014): 5.611

\section{Acknowledgement}

I would like to thank the anonymous referees for their helpful guidance that has improved the quality of this paper. Also I would like to thank my Project Guide Prof. P. M. Mane, for his valuable guidance.

\section{References}

[1] J. C. Bezdek and R. J. Hathaway, "Convergence of alternating optimization," J. Neural Parallel Scientific Comput., vol. 11, no. 4, pp. 351-368, 2003.

[2] C. C. Chang and C. J. Lin. (2004). Libsvm: A library for supportvector machines [Online]. Available:http://www.csie.ntu.edu.tw/cjlin/libsvm/

[3] G. Carenini, R. T. Ng, and E. Zwart, "Multi-document summarization of evaluative text," in Proc. ACL, Sydney, NSW, Australia, 2006, pp. 3-7.

[4] China Unicom 100 Customers iPhone User Feedback Report, 2009.

[5] ComScore Reports [Online]. Available:http://www.comscore.com/Press events/Press releases, 2011.

[6] X. Ding, B. Liu, and P. S. Yu, "A holistic lexicon-based approach to opinion mining," in Proc. WSDM, New York, NY, USA, 2008, pp. 231-240.

[7] G. Erkan and D. R. Radev, "LexRank: Graph-based lexical centrality as salience in text summarization," J. Artif. Intell. Res., vol. 22, no. 1, pp. 457-479, Jul. 2004.

[8] O. Etzioni et al., "Unsupervised named-entity extraction from the web: An experimental study," J. Artif. Intell., vol. 165, no. 1, pp. 91-134. Jun. 2005.

[9] A. Ghose and P. G. Ipeirotis, "Estimating the helpfulness and economic impact of product reviews: Mining text and reviewer characteristics," IEEE Trans. Knowl. Data Eng., vol. 23, no. 10, pp. 1498-1512. Sept. 2010.

[10] V. Gupta and G. S. Lehal, "A survey of text summarization extractive techniques," J. Emerg. Technol. Web Intell., vol. 2, no. 3, pp. 258-268, 2010.

[11] W. Jin and H. H. Ho, "A novel lexicalized HMM-based learning framework for web opinion mining," in Proc. 26th Annu. ICML, Montreal, QC, Canada, 2009, pp. 465-472.

[12] M. Hu and B. Liu, "Mining and summarizing customer reviews," in Proc. SIGKDD, Seattle, WA, USA, 2004, pp. $168-177$.

[13]K. Jarvelin and J. Kekalainen, "Cumulated gain-based evaluation of IR techniques," ACM Trans. Inform. Syst., vol. 20, no. 4, pp. 422-446, Oct. 2002.

[14] J. R. Jensen, "Thematic information extraction: Image classification," in Introductory Digit. Image Process., pp. 236-238.

[15]K. Lerman, S. Blair-Goldensohn, and R. McDonald, "Sentiment summarization: Evaluating and learning user preferences," in Proc. 12th Conf. EACL, Athens, Greece, 2009, pp. 514-522.

[16]F. Li et al., "Structure-aware review mining and summarization," in Proc. 23rd Int. Conf. COLING, Beijing, China, 2010, pp. 653-661.
[17]C. Y. Lin, "ROUGE: A package for automatic evaluation of summaries," in Proc. Workshop Text Summarization Branches Out, Barcelona, Spain, 2004, pp. 74-81.

[18]B. Liu, M. Hu, and J. Cheng, "Opinion observer: Analyzing and comparing opinions on the web," in Proc. 14th Int. Conf. WWW, Chiba, Japan, 2005, pp. 342351.

[19]B. Liu, "Sentiment analysis and subjectivity," in Handbook of Natural Language Processing, New York, NY, USA: Marcel Dekker, Inc., 2009.

[20]B. Liu, Sentiment Analysis and Opinion Mining. Mogarn \& Claypool Publishers, San Rafael, CA, USA, 2012.

[21]L. M. Manevitz and M. Yousef, "One-class SVMs for document classification," J. Mach. Learn., vol. 2, pp. 139-154, Dec. 2011.

[22] Q. Mei, X. Ling, M. Wondra, H. Su, and C. X. Zhai, "Topic sentiment mixture: Modeling facets and opinions in weblogs," in Proc. 16th Int. Conf. WWW, Banff, AB, Canada, 2007, pp. 171-180.

[23]B. Ohana and B. Tierney, "Sentiment classification of reviews using SentiWordNet," in Proc. IT\&T Conf., Dublin, Ireland, 2009.

[24] G. Paltoglou and M. Thelwall, "A study of information retrieval weighting schemes for sentiment analysis," inProc. 48th Annu. Meeting ACL, Uppsala, Sweden, 2010, pp. 1386-1395.

[25]B. Pang, L. Lee, and S. Vaithyanathan, "Thumbs up? Sentiment classification using machine learning techniques," in Proc. EMNLP, Philadelphia, PA, USA, 2002, pp. 79-86.

[26]B. Pang, L. Lee, and S. Vaithyanathan, "A sentimental education: Sentiment analysis using subjectivity summarization based on minimum cuts techniques," in Proc. ACL, Barcelona, Spain, 2004, pp. 271-278.

[27]B. Pang and L. Lee, "Opinion mining and sentiment analysis," in Found. Trends Inform. Retrieval, vol. 2, no. 1-2, pp. 1-135, 2008.

[28] A. M. Popescu and O. Etzioni, "Extracting product features and opinions from reviews," in Proc. HLT/EMNLP, Vancouver, BC, Canada, 2005, pp. 339346.

\section{Author Profile}

Harsha A. Patil Post graduate student of Zeal COE \& Research, Pune, Savitribai Phule Pune University. She received B.E. in Computer Engineering from North Maharashtra University, Jalgaon. Currently she is pursuing M.E. in Computer Engineering from Zeal COE \& Research Pune, Savitribai Phule Pune University.

Prof. P. M. Mane is currently working as Assistant Professor in Computer Engineering Department in Zeal COE \& Research Pune, Savitribai Phule Pune University. 